\title{
Retrospective Database Analysis: Dose Escalation and Adherence in Patients Initiating Biologics for Ulcerative Colitis
}

\author{
Millie D. Long ${ }^{a}$ Russell D. Cohen ${ }^{b}$ Timothy W. Smith ${ }^{c}$ Marco DiBonaventurac \\ David Gruben $^{d}$ Danielle Bargo ${ }^{c}$ Leonardo Salese ${ }^{\mathrm{e}}$ Daniel Quirk ${ }^{\mathrm{e}}$ \\ ${ }^{a}$ Center for Gastrointestinal Biology and Disease, University of North Carolina at Chapel Hill, Chapel Hill, NC, USA; \\ bInflammatory Bowel Disease Center, University of Chicago Medicine, Chicago, IL, USA; 'Pfizer Inc., New York, NY, \\ USA; dPfizer Inc., Groton, CT, USA; ePfizer Inc., Collegeville, PA, USA
}

\section{Keywords}

Biologic therapies - Dose escalation - Treatment

adherence . Ulcerative colitis

\begin{abstract}
Background: Biologic therapies are often used in patients with ulcerative colitis (UC) who are nonresponsive to conventional treatments. However, nonresponse or loss of response to biologics often occurs, leading to dose escalation, combination therapy, and/or treatment switching. We investigated real-world treatment patterns of biologic therapies among patients with UC in the USA. Methods: This study analyzed data from the IBM ${ }^{\circledR}$ MarketScan ${ }^{\circledR}$ Commercial and Medicare Supplemental Databases (medical/pharmacy claims for $>250$ million patients in the USA) to identify patients with UC initiating a biologic therapy (adalimumab, infliximab, golimumab, or vedolizumab) with 12 months of follow-up post-initiation. Key measures were patient baseline characteristics, dose escalation (average maintenance dose $>20 \%$ higher than label), adherence (proportion of days covered), and ulcerative colitis-related healthcare costs in the 12 months following biologic therapy initiation. $\boldsymbol{R e}$ sults: Of 2,331 patients included in the study (adalimumab $[N=1,291]$, infliximab $[N=810]$, golimumab $[N=127]$, and
\end{abstract}

vedolizumab [ $N=103]), 28.1 \%$ used concomitant immunosuppressant therapy within 12 months post-initiation. Overall, 23.6\% (adalimumab), 34.8\% (infliximab), 9.9\% (golimum$\mathrm{ab}$ ), and $39.2 \%$ (vedolizumab) of patients dose escalated within 12 months. Patients who dose escalated incurred USD 20,106 higher total UC-related healthcare costs over 12 months than those who did not. Adherence (covariate-adjusted proportion of days covered) ranged from 0.63 to 0.73 , and $39.3 \%$ of patients discontinued within 12 months (median treatment duration $=112$ days). Conclusion: Dose escalation was common, and incurred higher costs, in patients with UC initiating biologic therapies. Suboptimal adherence and/or discontinuation within 12 months of initiation occurred frequently, highlighting the challenges in managing these patients.

(c) 2021 The Author(s).

Published by S. Karger AG, Basel

\section{Introduction}

Ulcerative colitis (UC) is a chronic inflammatory disease of the colon that affects an estimated 910,000 people in the USA and is increasing in incidence and prevalence [1]. The primary clinical symptom is the presence of bloody diarrhea, which can be accompanied by a range of
C 2021 The Author(s).

Published by S. Karger AG, Basel

This is an Open Access article licensed under the Creative Commons Attribution-NonCommercial-4.0 International License (CC BY-NC) (http://www.karger.com/Services/OpenAccessLicense), applicable to the online version of the article only. Usage and distribution for commercial purposes requires written permission.
Correspondence to:

Leonardo Salese, leonardo.salese@pfizer.com 
other symptoms, including urgency and abdominal pain [2-5]. Individuals can also have more systemic symptoms such as fatigue, fever, and weight loss [2-4].

Treatment goals in UC focus on inducing clinical response via improvement in patient-reported outcomes, endoscopic improvement of inflammation, and maintenance of long-term, steroid-free remission [5]. For patients with mild to moderate disease severity, treatment typically consists of oral and/or rectal 5-aminosalicylates (5-ASA) for induction and maintenance of remission [5]. Systematic reviews and meta-analyses found that $29-46 \%$ of patients with UC who received 5-ASA entered remission [6], with $41-48 \%$ of patients then relapsing [7]. Approximately half of newly diagnosed patients progressed to moderately or severely active disease [8].

For patients with moderately to severely active disease who were intolerant to, or did not achieve maintenance of remission with, 5-ASA, the treatment options available at the time of this study (March 31,2017) were immunosuppressants (e.g., thiopurines) and several biologic therapies (adalimumab, infliximab, golimumab, and vedolizumab) $[5,9]$. Although these therapies are effective, not all patients respond, either failing to respond to induction therapy (primary nonresponse) or initially achieving remission but not maintaining it with long-term treatment (secondary loss of response). Among those who respond to biologic therapies, previous studies have indicated that $20-37 \%$ of patients lose response over time [10-13], which may at least partially be due to the formation of antidrug antibodies [14-16]. In the event of secondary loss of response, clinicians can choose to switch a patient's therapy to one with a different mechanism of action, or escalate the dose of their current biologic therapy $[5,17-19]$. At the time of this analysis, there were 3 approved biologic mechanisms of action for the treatment of moderate to severe UC (tumor necrosis factor inhibition, integrin receptor inhibition, and interleukin-12/23 inhibition) [20]. Investigating real-world treatment patterns could further our understanding of how frequently dose escalation or combination therapy are used with different biologic therapies in clinical practice. In addition, dose escalation has a substantial impact on pharmacy costs, since wholesale cost is proportional to increase in dose [19]. Therefore, real-world research could provide insights into the economic consequences of this practice [21], allowing evaluation of whether dose escalation is a cost-effective strategy.

The aim of this study was to understand the real-world treatment patterns of biologic therapies among patients with UC in the USA. The primary objectives were to as- sess the frequency of dose escalation (i.e., the use of doses or dosing frequencies higher than the labeled indication) and levels of adherence to therapy. Additional objectives were to assess the frequency of combination therapy (i.e., concomitant immunosuppressant and biologic therapy use) and possible reasons for discontinuation of biologic therapies, as available in administrative data.

\section{Patients and Methods}

\section{Data Source}

A retrospective cohort study was conducted using data from the IBM ${ }^{\circledR}$ MarketScan ${ }^{\circledR}$ Commercial and Medicare Supplemental Databases. The Commercial and Medicare Supplemental Databases consist of fully adjudicated medical (inpatient and outpatient diagnoses and procedures) and pharmacy (retail and mail order prescriptions) claims for $>250$ million patients across the USA. This study does not require Ethical Committee approval as it uses de-identified structured data.

\section{Sample}

The study included individuals who were commercially insured and/or had Medicare Supplemental insurance paid for by their employers, and who were treated with at least 1 biologic therapy (for the purposes of this study, the term "biologic therapy" referred specifically to biologic therapies indicated for the treatment of UC that were available at the time of the dataset: adalimumab, infliximab, golimumab, or vedolizumab) between January 01, 2012 and March 31, 2017, and who had at least 1 diagnosis code for UC (International Classification of Diseases, 9th revision [ICD-9] 556.X or International Classification of Diseases, 10th revision [ICD-10] K51.X [22, 23]) on or within 12 months prior to the initial claim for biologic therapy. Treatments approved for UC after March 31, 2017, were not included in this analysis. To be included in the final study sample, patients were required to have at least 12 months of continuous medical and pharmacy enrollment with the same insurer both prior to and after the date of their first use of that therapy (index date), and be between 18 and 89 years of age at the index date. Patients were included if they had no prescription for a biologic therapy indicated for UC in the 12-month pre-index period. Therefore, patients were either naïve to biologic therapy, or had not received biologic therapy for UC for at least 12 months prior to the index date. Patients were assumed to have moderately to severely active UC based on their physician's decision to prescribe a biologic therapy.

Patients were excluded from the study if they had any prescription for, or administration of, a second biologic therapy at index, or had a diagnosis of Crohn's disease (ICD-9 555.X or ICD-10 K50.X [22, 23]). Patients were categorized into treatment cohorts based on their index therapy (adalimumab, infliximab, golimum$\mathrm{ab}$, or vedolizumab).

\section{Demographic and Clinical Characteristics}

Demographic and clinical characteristics at index (age, sex, geographic region [Northeast, Midwest, South, and West] [24], and Quan-Charlson Comorbidity Index scores [calculated based on the presence of diagnosis codes in medical claims data]) [25], 
were collected. The 25 most common medications prescribed during the 12-month pre-index period were obtained, and those related to 5-ASA, steroids, or immunosuppressants are reported. Immunosuppressant use in the post-index period is also reported.

\section{Assessment of Dose Escalation}

Dose escalation was defined differently for each biologic therapy, although the principle was the same. First, an induction period was calculated for each therapy, based on the labeled induction length, with an extra 1 week added for adalimumab and golimumab, and an extra 2 weeks added for infliximab and vedolizumab, to account for slight delays or variation in administration. Induction doses for adalimumab (160 $\mathrm{mg}$ at week 0 , then $80 \mathrm{mg}$ at week 2), golimumab (200 $\mathrm{mg}$ at week 0 , then $100 \mathrm{mg}$ at week 2 ), and vedolizumab (300 $\mathrm{mg}$ at weeks 0,2 , and 6) were ignored for the purpose of calculating dose escalation (because doses, based on product labels, are higher for induction than during maintenance). If the average dose (i.e., accounting for both the dose and the frequency of treatment within this fixed time period) during maintenance (the 12-month post-index period, excluding the induction period) was at least $20 \%$ higher than the product label, this was considered dose escalation. For infliximab, since dosing is weight-based and weight was not available from the claims data, the index dose was used to derive an average daily baseline maintenance dose. This was calculated by dividing the total quantity administered at index by 56 (based on the labeled maintenance dose frequency of 8 weeks, or 56 days). If this estimated average dose during maintenance was at least $20 \%$ higher than the index dose, this was considered dose escalation. For all medications, dose escalation was evaluated as the only persistent portion of the maintenance period (treatment prior to a gap greater than 60 days) because the gap might signal a treatment interruption.

The proportion of patients with dose escalation during the 12 -month post-index period excluding the induction period (maintenance) was assessed. The effect of concomitant immunosuppressants on the proportion of patients with dose escalation was also explored. Additionally, all-cause direct medical costs paid out by insurance companies (inpatient, outpatient, and pharmacy) and UC-related costs paid out by insurance companies (inpatient and outpatient encounters with a diagnosis of UC, as well as physician-administered or prescriptions for UC-related drugs, including steroids, immunosuppressants, and biologic therapies) were calculated. Prescription drugs (self-administered) were found via pharmacy claims, whereas administrations (physician-administered) were found via medical claims. Infusible biologics could have been assigned differently depending on the patients' insurance plan; if they were part of the medical plan, they would comprise medical costs, and if they were part of the pharmacy plan, they would comprise pharmacy costs.

\section{Assessment of Adherence}

Adherence, defined as the extent to which a patient takes their medication as prescribed [26], was measured using a proportion of days covered (PDC) approach. This approach calculates the number of days the patient was treated (for prescriptions, days supplied was used; for administrations, treated days were imputed using the standard maintenance dosing schedule, regardless of patients' specific prescriptions) divided by the number of days in the 12 -month post-index period (a fixed denominator of 360 days), to arrive at a proportion. In order to prevent early prescription fills from impacting the calculation of adherence for self-administered biologic therapies, up to 15 days of stockpiling was incorporated by moving the early prescription fill date forward to coincide with the last day supplied by the previous prescription, rather than assuming that the biologic therapies were immediately administered. No stockpiling was used for physician-administered biologic therapies. A PDC $\geq 0.80$, a common threshold for evaluating medication adherence [26], was used as a cutoff to evaluate associations between characteristics and adherence. The same approach was used regardless of dose escalation.

\section{Statistical Analyses}

Data analyses were conducted in SAS v9.4 (Cary, NC, USA). Demographic and clinical characteristics, treatments, dose escalation, healthcare costs, and adherence are reported descriptively. Frequencies and percentages are reported for categorical variables, while means and standard deviations are reported for continuous variables. Where statistical testing across groups was applicable, $p$ values were generated from $\chi^{2}$ tests for the rates of the categorical variables, or $F$-tests for the means of continuous variables.

Treatment cohorts were compared with respect to adherence (defined dichotomously by a PDC $\geq 80$ vs. a PDC $<80$ ) using multivariable (generalized linear) models controlling for demographic and clinical characteristic differences, and specifying a binomial distribution and logit link function. Interactions between concomitant immunosuppressants and index therapies were also analyzed using these models, to predict dose escalation and adherence.

Variables thought to influence the dependent variables were compiled as a single set of confounding variables applied in all the subsequent multivariable models. All models controlled for age, sex, region, insurance type (Medicare vs. commercial insurance), health plan (health plan vs. employer), Quan-Charlson Comorbidity Index score, extent of disease based on ICD-9/ICD-10 codes, years to UC diagnosis during baseline, number of pre-index gastroenterology visits, number of pre-index UC hospitalizations, total pre-index UC-related healthcare costs, concomitant immunosuppressant (defined as azathioprine or 6-mercaptopurine) use, and index therapy. Adalimumab was selected as the reference cohort for the index therapy variable. Regression parameters, $95 \%$ confidence intervals, $\chi^{2}$ values, $p$ values, and adjusted probabilities (by index therapy only) are reported from these multivariable models.

\section{Results}

\section{Demographic and Clinical Characteristics}

A total of 2,331 patients were included in the study (Fig. 1), with most patients treated with adalimumab $(N$ $=1,291,55.4 \%)$, followed by infliximab $(N=810,34.7 \%)$, golimumab $(N=127,5.4 \%)$, and vedolizumab $(N=103$, $4.4 \%)$. Treatment cohort demographics were largely similar, although patients initiating vedolizumab were more likely to be male (58.3\%) compared with those initiating other treatments (50.4-51.5\%; Table 1).

The most common medications in the 12-month preindex period (prior to initiation of biologic therapy) are 
Fig. 1. Attrition during the determination of the study sample. $N$, number of patients. UC, ulcerative colitis. ${ }^{\text {aPatient data were }}$ drawn from a broader analysis of advanced therapies in UC, including biologic and immunosuppressant therapy. Patients initiating immunosuppressants only at index were excluded from this study.

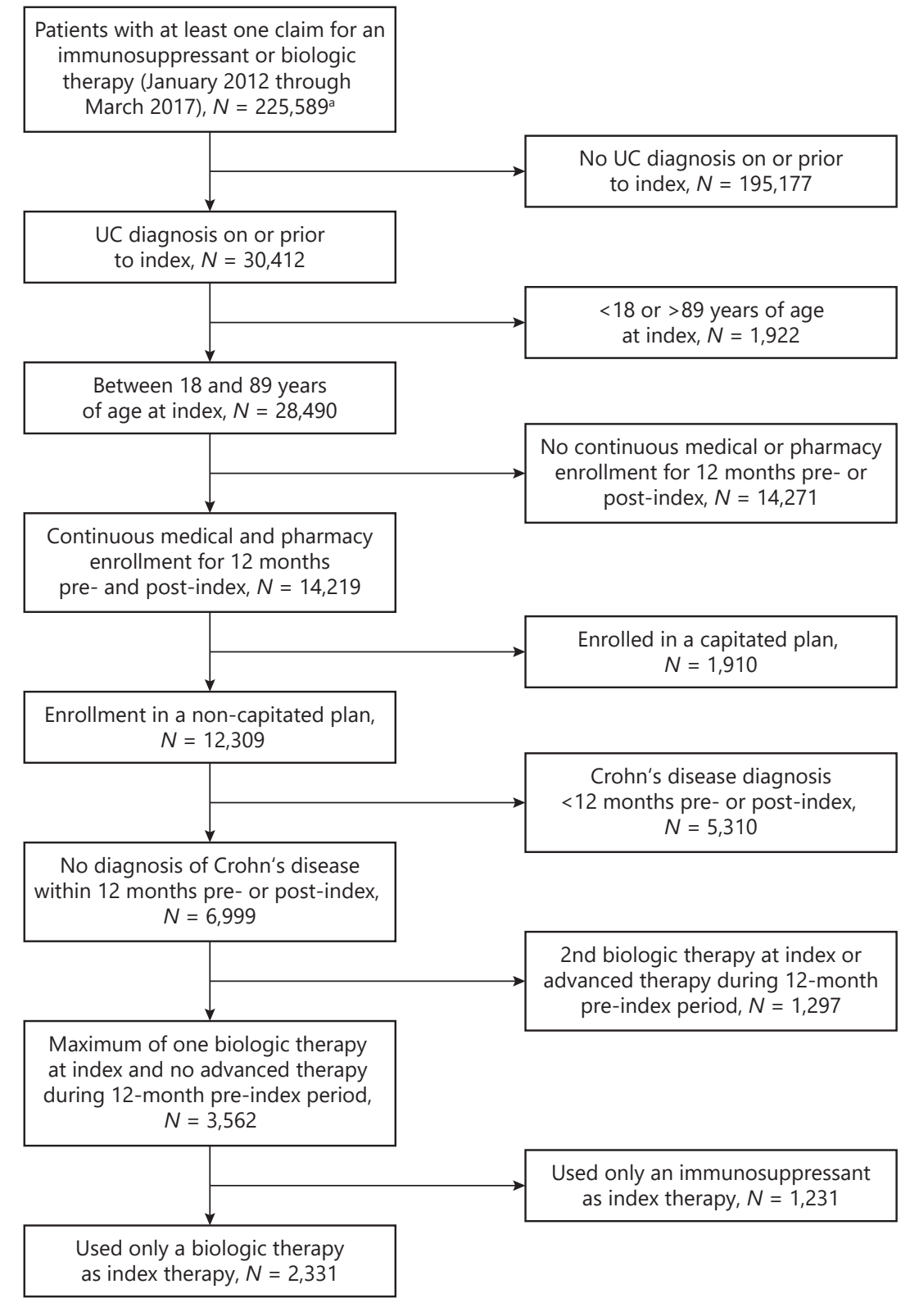

Patients with at least one claim for munosuppressant or biologic

UC diagnosis on or prior

to index, $N=195,177$

8 or $>89$ years of age at index, $N=1,922$

continuous medical or pharmacy post-index, $N=14,271$ summarized in Table 2. Treatment with 5-ASA, prednisone, and/or other steroids and immunosuppressants occurred frequently (Table 2).

During the 12-month post-index period (following initiation of biologic therapy), combination therapy was common, with $27.9 \%$ of adalimumab-treated patients, $29.6 \%$ of infliximab-treated patients, $26.0 \%$ of golimumab-treated patients, and $21.4 \%$ of vedolizumab-treated patients also using an immunosuppressant.

\section{Dose Escalation}

Dose escalation was examined among patients with at least 1 administration of their index therapy during maintenance (since dose escalation was only examined in maintenance). Of these patients $(N=1,831), 1,335(72.9 \%)$ were classified as not having a dose escalation and 496 (27.1\%) were classified as having a dose escalation. Dose escalation was highest in patients initiating infliximab or vedolizumab (Fig. 2). 
Table 1. Demographic and clinical characteristics at index, by treatment cohort

\begin{tabular}{|c|c|c|c|c|c|c|}
\hline Male, $n(\%)$ & $1,200(51.5)$ & $659(51.0)$ & $417(51.5)$ & $64(50.4)$ & $60(58.3)$ & 0.560 \\
\hline Age, mean (SD), years & $44.5(15.0)$ & $44.8(14.4)$ & $43.8(15.8)$ & $45.3(15.2)$ & $44.4(15.7)$ & 0.455 \\
\hline \multicolumn{7}{|l|}{ Region, $n(\%)$} \\
\hline Northeast & 407 (17.5) & $229(17.7)$ & $132(16.3)$ & $30(23.6)$ & $16(15.5)$ & \\
\hline Midwest & $560(24.0)$ & $278(21.5)$ & $240(29.6)$ & $20(15.7)$ & $22(21.4)$ & \\
\hline West & $377(16.2)$ & $218(16.9)$ & $120(14.8)$ & $18(14.2)$ & $21(20.4)$ & \\
\hline Unknown & $18(0.8)$ & $11(0.9)$ & $6(0.7)$ & $1(0.8)$ & $0(0.0)$ & \\
\hline Quan-Charlson Comorbidity Index score, mean (SD) & $0.6(1.0)$ & $0.6(1.1)$ & $0.6(1.1)$ & $0.6(1.3)$ & $0.4(0.9)$ & 0.172 \\
\hline
\end{tabular}

$N$, number of patients; $n$, number of patients within the given category; SD, standard deviation. $p$ values are from $x^{2}$ tests for the rates of the categorical variables, or an F-test for the means of continuous variables, comparing the 4 biologic therapies.

Table 2. The most common UC-related medications during the 12-month pre-index period, by treatment cohort

\begin{tabular}{|c|c|c|c|c|c|c|}
\hline \multicolumn{7}{|l|}{ 5-ASA, $n(\%)$} \\
\hline Mesalamine & $1,728(74.1)$ & $983(76.1)$ & $578(71.4)$ & $95(74.8)$ & 72 (69.9) & 0.073 \\
\hline \multicolumn{7}{|l|}{ Steroids, $n(\%)$} \\
\hline Budesonide & $665(28.5)$ & $372(28.8)$ & $216(26.7)$ & $45(35.4)$ & $32(31.1)$ & 0.193 \\
\hline Hydrocortisone & $538(23.1)$ & $291(22.5)$ & $188(23.2)$ & $43(33.9)$ & $16(15.5)$ & 0.008 \\
\hline Prednisone & $1,647(70.7)$ & $929(72.0)$ & $584(72.1)$ & $83(65.4)$ & $51(49.5)$ & $<0.001$ \\
\hline \multicolumn{7}{|l|}{ Immunosuppressants, $n$ (\%) } \\
\hline Azathioprine/6-mercaptopurine & $655(28.1)$ & $360(27.9)$ & $240(29.6)$ & $33(26.0)$ & $22(21.4)$ & 0.312 \\
\hline
\end{tabular}

Total all-cause and UC-related costs were significantly higher overall (USD 19,662 and USD 20,106 higher, respectively) in patients who had a dose escalation in the 12-month post-index period compared with those who did not (Table 3). Total all-cause costs were higher in patients who had a dose escalation on adalimumab, infliximab, or vedolizumab than in those who did not. Patients who had a dose escalation on adalimumab, infliximab, or vedolizumab had total UC-related costs that were USD 22,247 , USD 16,287, and USD 47,734 higher, respectively, than those for patients who did not have a dose escalation on these treatments (all $p<0.05$ ). Dose escalation in patients using golimumab was uncommon (Fig. 2), and the total all-cause and UC-related costs in the 12-month post-index period among patients using golimumab were

Dose Escalation and Adherence to

Biologics in UC
USD 9,922 and USD 7,758 less, respectively, for patients who had a dose escalation $(N=10)$ compared with those who did not $(N=91)$.

UC-related inpatient and outpatient costs were not significantly different ( $p=0.98$ and $p=0.20$, respectively) between patients who did or did not have a dose escalation (Table 3). UC-related prescription and drug administration costs were higher among those who had a dose escalation than those who did not (both $p<0.05$ ). Large differences were also seen between UC-related prescription and drug administration costs. For adalimumab and golimumab, prescription costs were much higher than drug administration costs, and for infliximab and vedolizumab, drug administration costs were much higher than prescription costs. 
Fig. 2. Percentage of patients who had a dose escalation (i.e., received an average daily dose $>20 \%$ higher than specified in the label) during the maintenance phase of the 12-month post-index period, by treatment cohort.

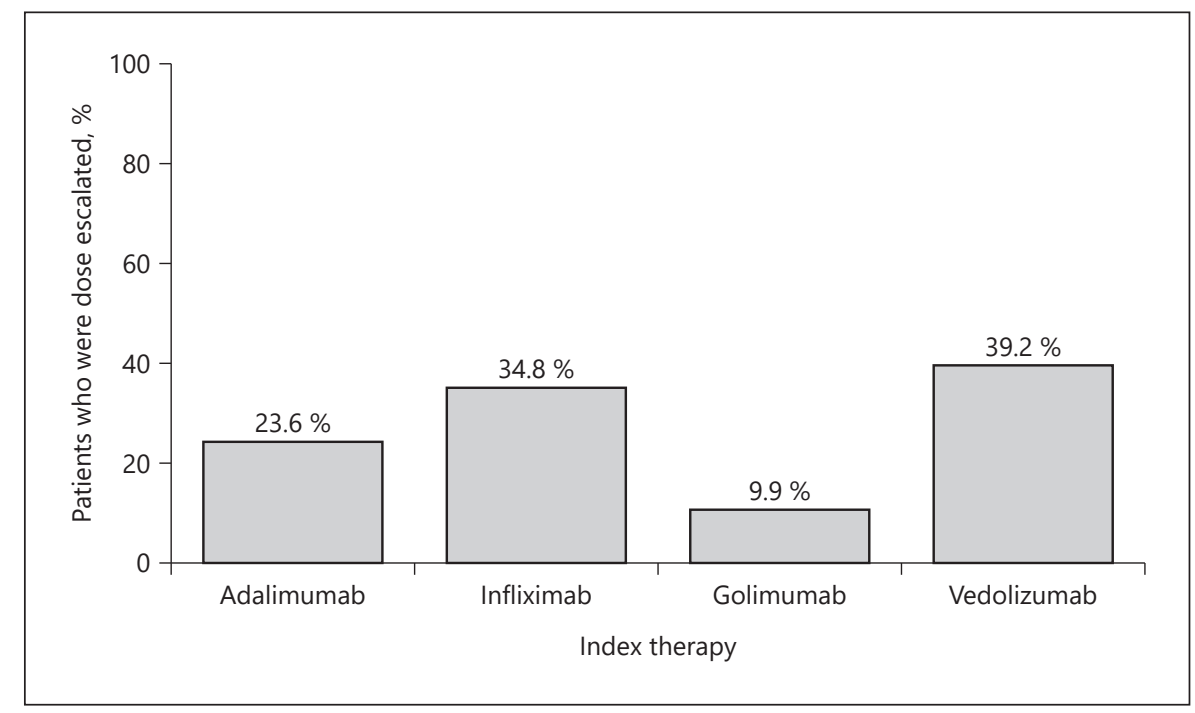

The effect of concomitant immunosuppressants on the proportion of patients with dose escalation was also assessed (Fig. 3). Across index therapies, the proportion of patients with dose escalation was largely similar among those receiving and not receiving concomitant immunosuppressants. In all treatment groups, except for golimumab, the proportion of patients with dose escalation appeared numerically higher among patients who received concomitant immunosuppressants than among those who did not. However, a multivariable model assessing the interaction between immunosuppressants and index therapy to predict dose escalation did not reveal any significant interactions (data not shown). The data from this model suggest that using concomitant immunosuppressants did not affect dose escalation.

\section{Adherence}

Adherence, measured by PDC, was as follows: overall, 0.71; adalimumab, 0.68; infliximab, 0.76; golimumab, 0.67 ; and vedolizumab, 0.77 . Overall, the proportion of adherent patients (those achieving a PDC $\geq 0.80$ ) was $56.2 \%$. A PDC $\geq 0.80$ was achieved by $51.9 \%$ of patients treated with adalimumab and $55.1 \%$ of patients treated with golimumab, both of which were administered subcutaneously $[27,28]$. By comparison, a PDC $\geq 0.80$ was achieved by $62.5 \%$ and $63.1 \%$ of patients treated with the intravenous infusion therapies $[29,30]$ infliximab and vedolizumab, respectively.

A similar pattern was observed after adjusting for patient characteristic variables (age, sex, region, insurance type, health plan, Quan-Charlson Comorbidity Index score, extent of disease, years to UC diagnosis during baseline, number of pre-index gastroenterology visits, number of pre-index UC hospitalizations, total pre-index UC-related healthcare costs, concomitant immunosuppressant use, and index therapy). The adjusted PDC was 0.63 for patients treated with golimumab and 0.65 for patients treated with adalimumab. Compared with the adalimumab cohort, PDC for the infliximab and vedolizumab cohorts was significantly higher at 0.73 and 0.71 , respectively (both $p<0.05$ ). Analysis of predictors of adherence defined as a PDC $\geq 80$ is shown in online supplementary Table 1 (for all online suppl. material, see www. karger.com/doi/10.1159/000521299). Being from the Midwest region (vs. the Northeast region; odds ratio = 1.33), having longer disease duration (odds ratio $=1.18$ ), and having more gastroenterology visits during the preindex period (odds ratio $=1.04$ ) were other factors associated with greater adherence (all $p<0.05$ ). Having a higher number of pre-index UC-related hospitalizations (odds ratio $=0.83 ; p<0.05$ ) was associated with lower adherence. A multivariable model assessing the interaction between immunosuppressants and index therapy to predict adherence did not reveal any significant interactions, suggesting that the use of concomitant immunosuppressants did not affect the likelihood of patients being adherent to their index therapy.

\section{Discussion}

This study explored dose escalation and treatment adherence over a 12 -month period in patients with UC initiating biologic therapies in the USA. In this study, dose 
Table 3. All-cause and UC-related costs by dose escalation

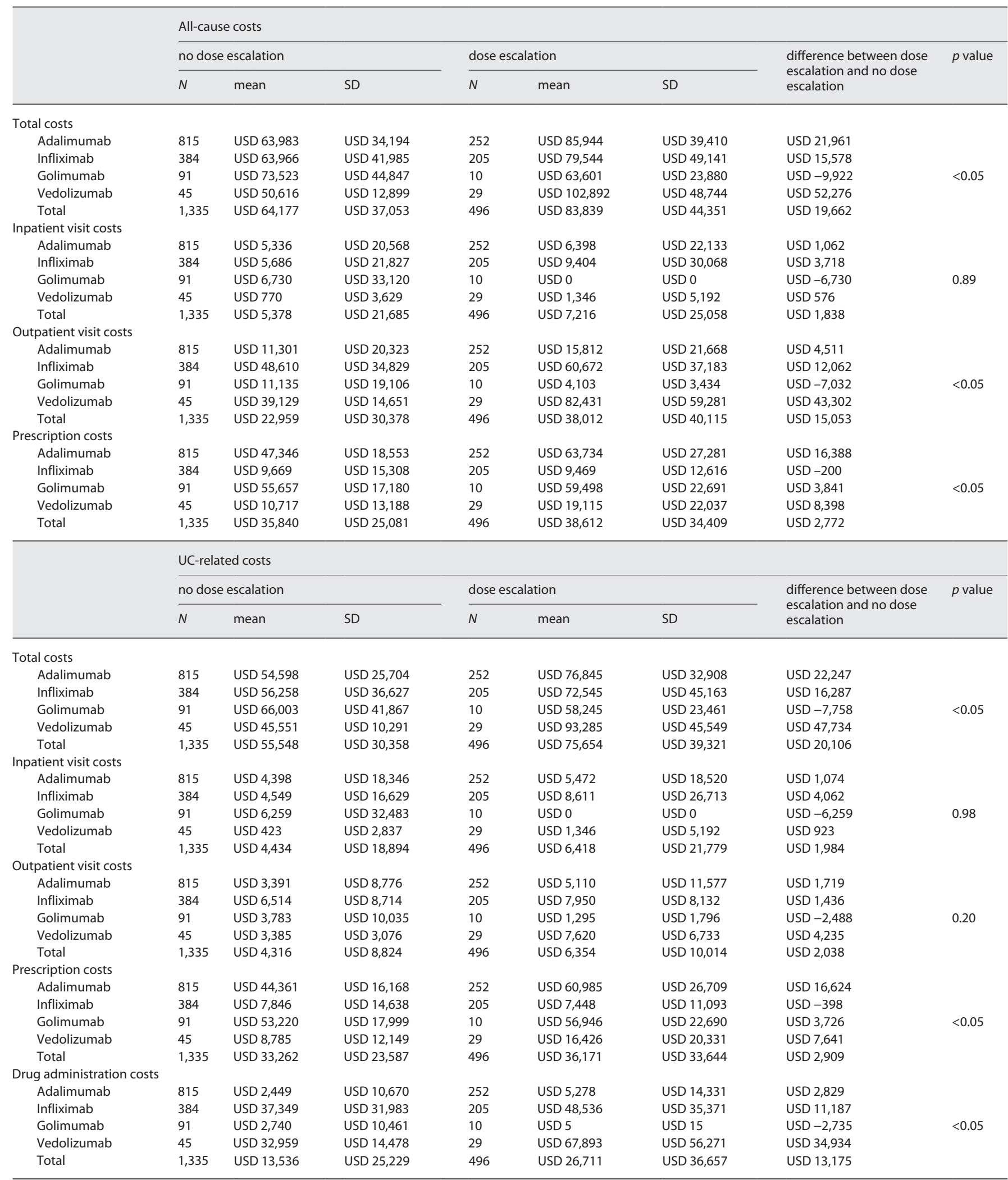

$N$, number of patients; SD, standard deviation; UC, ulcerative colitis. $p$ values represent the statistical testing of the main effect of dose escalation on each cost variable; costs are broken out by index therapy only for descriptive purposes.

Dose Escalation and Adherence to

Biologics in UC
Dig Dis 2022;40:553-564

DOI: $10.1159 / 000521299$ 


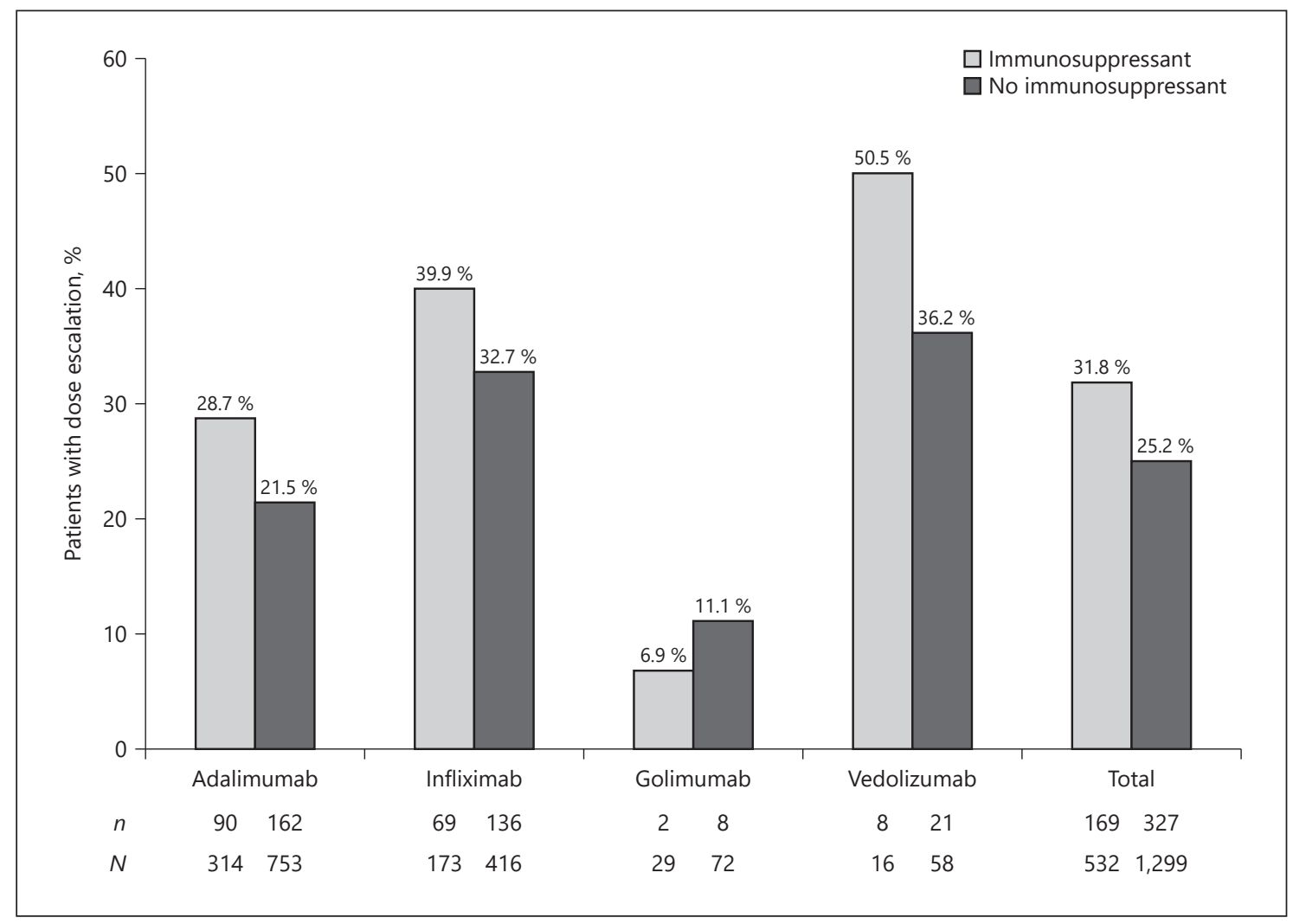

Fig. 3. The proportion of patients who had a dose escalation 12 months post-index, by concomitant immunosuppressants and index treatment. $N$, total number of patients in which dose escalation was examined; $n$, number of patients in each group (patients with dose escalation; patients receiving concomitant immunosuppressants; or not receiving concomitant immunosuppressants). The 25 most common concomitant medications were obtained.

escalation was observed in up to $39.2 \%$ of patients in the first year after initiating biologic therapies, and incurred USD 20,106 more in total UC-related annual costs than in patients who did not have a dose escalation. Overall, the proportion of adherent patients was $56.2 \%$. The proportion of adherent patients was around $10 \%$ higher in patients initiating intravenous biologic therapies than in those initiating subcutaneous biologic therapies. Concomitant immunosuppressants were used in $29.1 \%$ of the 1,831 patients who were examined for dose escalation. In all treatment groups, except for golimumab, the proportion of patients with dose escalation was numerically higher in patients who received concomitant immunosuppressants than in those who did not. This is an interesting finding because treatment with biologics in combination with immunosuppressants, particularly infliximab and thiopurines, has been observed as superior to biologic monotherapy in patients with moderately to se- verely active UC [31]. However, multivariable modeling assessing the interaction between immunosuppressants and index therapy to predict dose escalation did not reveal any significant interactions. However, this result should be interpreted with caution because patients being treated with concomitant immunosuppressants may be more likely to have a dose escalation in some cases, e.g., in patients with low serum drug trough concentration and borderline antidrug antibody formation to biologics [32]. It is also possible that this group of patients had more severe disease at baseline, thus requiring a concomitant immunosuppressant alongside their biologic therapy.

The rates of dose escalation observed in this study may reflect the challenges in achieving remission in many patients initiating biologic therapies, indicating that currently approved doses may be insufficient to control UC in some patients. Interestingly, recent randomized con- 
trolled trial data have demonstrated that dose intensification of adalimumab was not associated with improved endoscopic or clinical outcomes after induction [33]. There are also economic considerations to dose escalation. Patients who had a dose escalation incurred higher total annual costs, based on increased pharmacy and administration costs, although this varied by therapy. This cost increase for patients who had a dose escalation suggests that total all-cause and UC-related costs are proportional to the dose or dose frequency used. Prescription costs were much higher than drug administration costs for adalimumab and golimumab, whereas drug administration costs were much higher than prescription costs for infliximab and vedolizumab. This is likely due to the fact that adalimumab and golimumab are self-administered, so costs would be recorded under prescription claims, whereas infliximab and vedolizumab are physician-administered, so costs would be recorded under medical claims.

The study demonstrated that adherence, measured by PDC, was higher for intravenous infusion therapies (infliximab and vedolizumab) than for subcutaneous therapies (adalimumab and golimumab), suggesting that adherence is better in patients who attend scheduled appointmentstoreceivephysician-administeredintravenous therapy, compared with those who self-administer subcutaneous treatment. However, overall, 36.9-48.1\% of patients were nonadherent (with adherence defined as a PDC $\geq 0.80$ ). These real-world data reinforce the pervasive problem of nonadherence, which is often underestimated by clinicians in inflammatory bowel disease treatment [34]. Multivariable analysis demonstrated that patients with longer disease duration and more regular contact with their gastroenterologists were less at risk of being nonadherent. Longer times between gastroenterologist visits (at least 3 months) have previously been associated with nonadherence [35]. It is possible that patients with more severe disease have more contact with their gastroenterologist and, as such, may be more adherent than patients with less severe disease who have less frequent contact with their gastroenterologist. Multivariable analysis also identified an association between UC hospitalizations and lower rates of adherence, suggesting that patients with a history of UC hospitalizations could also be more at risk of being nonadherent. However, the increase in hospitalizations could be a result of nonadherence due to poorer disease control. It is difficult to speculate on these results because hospitalizations may have interrupted patients' treatment schedules. Hospitalizations may also have confounded adherence results be-

Dose Escalation and Adherence to Biologics in UC cause they are associated with a more aggressive disease course and increased risk of colectomy [36]. Other etiologies for nonadherence, such as concurrent infection where physicians recommend holding therapy, which leads to a delayed treatment schedule, can be difficult to determine from administrative claims data. An alternate indication for discontinuation of therapy is colectomy; in this study, however, nonadherence was defined by the patients' prescription record only. More research is necessary to clarify the factors that impact adherence.

This study was subject to several additional limitations due to the nature of the analyses. Outpatient administrations of biologic therapy were included in these analyses; however, IBM ${ }^{\circledR}$ MarketScan ${ }^{\circledR}$ Commercial and Medicare Supplemental Databases did not contain data on inpatient medication administration; therefore, administration of biologic therapy during an inpatient stay could not be determined. Real-world dosing and administration is complex, and a set of simplifying assumptions therefore had to be imposed for research purposes. A different set of assumptions (e.g., for dose escalation) may yield different results. Multivariable modeling controlled for observed differences in demographic and clinical characteristics, but some issues may be associated with this. For example, extent of disease was included as a covariate in the models, based on ICD-9/10 codes that have been associated with a large degree of variability. Despite this variability, the inclusion of extent of disease would not have influenced the models. Another potential limitation with multivariable modeling is that there may be other variables, not included in the data, which may explain some of the variation among treatment cohorts. For example, replacement shipments and refrigeration issues were not taken into account when performing average dose escalation calculations. Additionally, patients treated with infliximab might have experienced weight gain, leading to an increase in dose (as dosing is weight-based) that could have resulted in an overrepresentation of the frequency of dose escalation in these patients. Weight was not available in the claims data, so this could not be verified. Furthermore, patients who were initiated on doses of infliximab $>20 \%$ higher than the product label and maintained on these doses would not be considered in this study to have dose escalated; however, such patients may be expected to generate higher costs from higher doses.

Another limitation of this study is that the duration of evaluation was limited to 12 months following initiation, and these findings cannot be extrapolated to later years where dose escalation may be higher. Patients were only excluded if they had received a biologic therapy up to 12 
months before the index period; therefore, they may have received a biologic therapy prior to the pre-index period and hence would not be considered truly biologic-naïve. Moreover, the sample used for this study comprised patients with UC, newly treated with a biologic therapy, who were continuously enrolled in a commercial health insurance plan. Therefore, the findings reported here may not be generalizable to other populations, such as uninsured patients.

Patient numbers were not evenly weighted across treatment cohorts. Adalimumab was the most common index therapy, with $1,291 / 2,331$ (55.4\%) patients in this treatment cohort. Switching to a treatment with a different mechanism of action following secondary loss of response only became an option when vedolizumab was approved in the USA in 2014 [30]. Since this study included data from 2012 to 2017 , there could be an overrepresentation of the number of patients that had a dose escalation compared with current clinical practice.

Additionally, patients were assumed to have moderately to severely active UC based on the fact that their physician prescribed a biologic therapy. However, the study may have excluded patients who had UC alongside another inflammatory disease and were treated primarily for the other condition, and it may have included patients with UC who had a concomitant diagnosis of another inflammatory condition, such as rheumatoid arthritis, ankylosing spondylitis, psoriasis, or psoriatic arthritis, whose prescriptions for the biologic therapy were intended to treat these other comorbidities.

Patients who underwent colectomy prior to index therapy were not excluded from this study; therefore, it is possible that the analysis population included some patients who were receiving therapy for post-colectomy pouchitis. However, it is expected that this would represent only a very small proportion of the overall patient population in this study.

Finally, patients who filled their prescription but did not take their biologic therapy could not be accounted for when measuring adherence and patients who were considered to have discontinued their biologic therapy may actually have bridged the gap between biologics with steroids, leading to possible misrepresentation of adherence. Measurement of adherence could also have been affected by the administration method of the biologic therapy, because only medication possession could be recorded in patients prescribed self-administered injectables (adalimumab and golimumab), whereas patients prescribed physician-administered intravenous biologic therapies (infliximab and vedolizumab) were known to have received the drug.

\section{Conclusions}

In this study of patients initiating biologic therapies for the treatment of moderately to severely active UC, who were followed for 12 months, up to $39.2 \%$ of patients had a dose escalation within the first year, resulting in up to USD 47,734 higher UC-related costs compared with patients who did not have a dose escalation. Furthermore, suboptimal adherence was observed in the 12-month post-index period. The treatment patterns observed in this study suggest that new treatment options offering additional mechanisms of action, dosing regimens, and/or other characteristics should be pursued in patients with moderately to severely active UC.

\section{Acknowledgments}

Medical writing support, under the guidance of the authors, was provided by Nina Divorty, $\mathrm{PhD}$, and Molly MacFadyen, MSc, CMC Connect, McCann Health Medical Communications and was funded by Pfizer Inc, New York, NY, USA, in accordance with Good Publication Practice (GPP3) guidelines (Ann Intern Med. 2015;163:461-464).

\section{Statement of Ethics}

In compliance with the Health Insurance Portability and Accountability Act, patient data included in the study were de-identified. As a retrospective analysis of de-identified data, Institutional Review Board review was not required or sought for this study and written informed consent from participants was not required in accordance with local/national guidelines.

\section{Conflict of Interest Statement}

M.D.L. has received consultancy fees from AbbVie, Janssen, Pfizer Inc., Takeda, Target PharmaSolutions, and Theravance, and has received grant support from Pfizer Inc and Takeda. R.D.C. has received speaker fees from AbbVie and Takeda and has received consultancy fees from AbbVie, Celgene, Janssen, Pfizer Inc., and Takeda. T.W.S. and D.B. were employees of Pfizer Inc. at the time this research was conducted. M.D.B., D.G., L.S., and D.Q. are employees and stockholders of Pfizer Inc.

\section{Funding Sources}

This work was supported by Pfizer Inc. The study was sponsored by Pfizer Inc. 


\section{Author Contributions}

M.D.L., R.D.C., T.W.S., M.D.B., D.G., D.B., L.S., and D.Q. contributed to the manuscript's conception and design and critically reviewed it. All authors read and approved the final manuscript.

\section{Data Availability Statement}

Upon request, and subject to review, Pfizer will provide the data that support the findings of this study. Subject to certain criteria, conditions, and exceptions, Pfizer may also provide access to the related individual de-identified participant data. See https:// www.pfizer.com/science/clinical-trials/trial-data-and-results for more information.

\section{References}

1 Shivashankar R, Tremaine WJ, Harmsen WS, Loftus EV Jr. Incidence and prevalence of Crohn's disease and ulcerative colitis in Olmsted County, Minnesota from 1970 to 2010 Clin Gastroenterol Hepatol. 2017 Jun;15(6): $857-63$.

2 Danese S, Fiocchi C. Ulcerative colitis. N Engl J Med. 2011 Nov;365(18):1713-25.

3 Mowat C, Cole A, Windsor A, Ahmad T, Arnott I, Driscoll R, et al. Guidelines for the management of inflammatory bowel disease in adults. Gut. 2011 May;60(5):571-607.

4 Fakhoury M, Negrulj R, Mooranian A, Al-Salami $\mathrm{H}$. Inflammatory bowel disease: clinical aspects and treatments. J Inflamm Res. 2014 Jun; 7:113-20.

5 Rubin DT, Ananthakrishnan AN, Siegel CA, Sauer BG, Long MD. ACG clinical guideline: ulcerative colitis in adults. Am J Gastroenterol. 2019 Mar;114(3):384-413.

6 Wang Y, Parker CE, Bhanji T, Feagan BG, MacDonald JK. Oral 5-aminosalicylic acid for induction of remission in ulcerative colitis. Cochrane Database Syst Rev. 2016 Apr;4: CD000543.

7 Wang Y, Parker CE, Feagan BG, MacDonald JK. Oral 5-aminosalicylic acid for maintenance of remission in ulcerative colitis. Cochrane Database Syst Rev. 2016 May; 5: CD000544

8 Solberg IC, Lygren I, Jahnsen J, Aadland E, Høie O, Cvancarova M, et al. Clinical course during the first 10 years of ulcerative colitis: results from a population-based inception cohort (IBSEN Study). Scand J Gastroenterol. 2009;44(4):431-40.

9 Dubinsky MC. Reviewing treatments and outcomes in the evolving landscape of ulcerative colitis. Postgrad Med. 2017 Jun;129(5): 538-53.

10 Reinisch W, Sandborn WJ, Rutgeerts P, Feagan BG, Rachmilewitz D, Hanauer SB, et al. Long-term infliximab maintenance therapy for ulcerative colitis: the ACT-1 and - 2 extension studies. Inflamm Bowel Dis. 2012 Feb; 18(2):201-11.

11 Sandborn WJ, Feagan BG, Marano C, Zhang H, Strauss R, Johanns J, et al. Subcutaneous golimumab maintains clinical response in patients with moderate-to-severe ulcerative colitis. Gastroenterology. 2014 Jan;146(1):96-109.
12 Loftus EV Jr, Colombel JF, Feagan BG, Vermeire S, Sandborn WJ, Sands BE, et al. Longterm efficacy of vedolizumab for ulcerative colitis. J Crohns Colitis. 2017 Apr;11(4):40011.

13 Taxonera C, Iglesias E, Muñoz F, Calvo M, Barreiro-de Acosta M, Busquets D, et al Adalimumab maintenance treatment in ulcerative colitis: outcomes by prior anti-TNF use and efficacy of dose escalation. Dig Dis Sci. 2017 Feb;62(2):481-90.

14 Steenholdt C, Al-khalaf M, Brynskov J, Bendtzen K, Thomsen OØ, Ainsworth MA. Clinical implications of variations in anti-infliximab antibody levels in patients with inflammatory bowel disease. Inflamm Bowel Dis. 2012 Dec; 18(12):2209-17.

15 Nanda KS, Cheifetz AS, Moss AC. Impact of antibodies to infliximab on clinical outcomes and serum infliximab levels in patients with inflammatory bowel disease (IBD): a metaanalysis. Am J Gastroenterol. 2013 Jan;108(1): $40-7$

16 Roblin X, Marotte H, Leclerc M, Del Tedesco E, Phelip JM, Peyrin-Biroulet L, et al. Combination of C-reactive protein, infliximab trough levels, and stable but not transient antibodies to infliximab are associated with loss of response to infliximab in inflammatory bowel disease. J Crohns Colitis. 2015 Jul;9(7): 525-31.

17 Kornbluth A, Sachar DB, Practice Parameters Committee of the American College of Gastroenterology. Ulcerative colitis practice guidelines in adults: American college of gastroenterology, practice parameters committee. Am J Gastroenterol. 2010 Mar;105(3): 501-23.

18 Park SC, Jeen YT. Current and emerging biologics for ulcerative colitis. Gut Liver. 2015 Jan;9(1):18-27.

19 Shahwan KT, Kimball AB. Managing the dose escalation of biologics in an era of cost containment: the need for a rational strategy. Int J Womens Dermatol. 2016 Oct;2(4):151-3.

20 Sabino J, Verstockt B, Vermeire S, Ferrante M. New biologics and small molecules in inflammatory bowel disease: an update. Therap Adv Gastroenterol. 2019 May; 12: 1756284819853208 .
21 Black CM, Yu E, McCann E, Kachroo S. Dose escalation and healthcare resource use among ulcerative colitis patients treated with adalimumab in English hospitals: an analysis of realworld data. PLoS One. 2016 Feb;11(2): e0149692.

22 Centers for Disease Control and Prevention. International classification of diseases, ninth revision, clinical modification (ICD9-CM); 2015. December 09, 2020. Available from: http: //www.cdc.gov/nchs/icd/icd$9 \mathrm{~cm} . \mathrm{htm}$.

23 Centers for Disease Control and Prevention. International classification of diseases, tenth revision, clinical modification (ICD-10-CM); 2018. December 09, 2020. Available from: https://www.cdc.gov/nchs/icd/icd10 cm.htm.

24 United States Census Bureau. U.S. and world population clock. 2020. December 09, 2020. Available from: https://www.census.gov/ popclock/.

25 Quan H, Li B, Couris CM, Fushimi K, Graham $\mathrm{P}$, Hider $\mathrm{P}$, et al. Updating and validating the Charlson comorbidity index and score for risk adjustment in hospital discharge abstracts using data from 6 countries. Am J Epidemiol. 2011 Mar;173(6): 676-82.

26 Osterberg L, Blaschke T. Adherence to medication. N Engl J Med. 2005 Aug;353(5):48797.

27 Janssen Biotech Inc. SIMPONI ${ }^{\circledR}$ (golimumab): highlights of prescribing information. 2019. December 09, 2020. Available from: https: //www.janssenmd.com/pdf/simponi/ simponi_pi.pdf.

28 AbbVie Inc. HUMIRA ${ }^{\circledR}$ (adalimumab): highlights of prescribing information; 2020. December 09, 2020. Available from: https:// www.rxabbvie.com/pdf/humira.pdf.

29 Janssen Biotech Inc. REMICADE ${ }^{\circledR}$ (infliximab): highlights of prescribing information; 2020. December 09, 2020. Available from: http: //www.janssenlabels.com/package-insert/product-monograph/prescribing-information/REMICADE-pi.pdf.

30 Takeda Pharmaceuticals America Inc. ENTYVIO $^{\circledR}$ (vedolizumab): highlights of prescribing information; 2020. December 09, 2020. Available from: https://general.takedapharm.com/ENTYVIOPI. 
31 Panaccione R, Ghosh S, Middleton S, Márquez JR, Scott BB, Flint L, et al. Combination therapy with infliximab and azathioprine is superior to monotherapy with either agent in ulcerative colitis. Gastroenterology. 2014 Feb; 146(2):392-400.e3.

32 Lichtenstein GR. Comprehensive review: antitumor necrosis factor agents in inflammatory bowel disease and factors implicated in treatment response. Therap Adv Gastroenterol. 2013 Jul;6(4):269-93.
33 Colombel JF, Panés J, D’Haens G, Schreiber S, Panaccione R, Peyrin-Biroulet L, et al. OP01 Higher versus standard adalimumab maintenance regimens in patients with moderately to severely active ulcerative colitis: results from the SERENE-UC maintenance study (abstract). J Crohns Colitis. 2020 Jan;14(1): S001.

34 Soobraty A, Boughdady S, Selinger CP. Current practice and clinicians' perception of medication non-adherence in patients with inflammatory bowel disease: a survey of 98 clinicians. World J Gastrointest Pharmacol Ther. 2017 Feb;8(1):67-73.
35 Tae CH, Jung SA, Moon HS, Seo JA, Song HK, Moon CM, et al. Importance of patients knowledge of their prescribed medication in improving treatment adherence in inflammatory bowel disease. J Clin Gastroenterol. 2016 Feb;50(2):157-62.

36 Ananthakrishnan AN, Issa M, Beaulieu DB, Skaros S, Knox JF, Lemke K, et al. History of medical hospitalization predicts future need for colectomy in patients with ulcerative colitis. Inflamm Bowel Dis. 2009 Feb;15(2):17681. 